\title{
Erratum to: Evaluation of the ESUR PI-RADS scoring system for multiparametric MRI of the prostate with targeted MR/TRUS fusion-guided biopsy at 3.0 Tesla
}

\author{
M. C. Roethke • T. H. Kuru • S. Schultze • D. Tichy • \\ A. Kopp-Schneider • M. Fenchel • H.-P. Schlemmer • \\ B. A. Hadaschik
}

Published online: 7 February 2014

(C) European Society of Radiology 2014

\section{Erratum to: Eur Radiol}

$$
\text { DOI 10.1007/s00330-013-3017-5 }
$$

While reviewing our article above, which was published online on Oct, 3rd it has come to our attention that a few isolated numbers were inadvertently switched during our revision of the manuscript:

"Sensitivity/Specificity for the following thresholds were calculated: $73 \% / 92 \%$ and $85 \% / 67 \%$ for PI-RADS scores of 9 and 10 , respectively; $85 \% / 56 \%$ and $60 \% / 97 \%$ for Likert scores of 3 and 4 , respectively."

The correct version (as per the results in Table 7) should have read:

"Sensitivity/Specificity for the following thresholds were calculated: $85 \% / 73 \%$ and $67 \% / 92 \%$ for PI-RADS scores of 9 and 10, respectively; $85 \% / 60 \%$ and $56 \% / 97 \%$ for Likert scores of 3 and 4 , respectively."
In Table 5: 5th row, line 3: it should have read "78.6" instead of " 7.86 "

Finally, four lines above "Discussion", last section: it should have read "DCE" instead of "DCI"

As corresponding author I deeply regret this circumstance and accept full responsibility for this error. We deeply apologize for any inconveniences this may cause.

The online version of the original article can be found at http:// dx.doi.org/10.1007/s00330-013-3017-5.

M. C. Roethke $(\bowtie) \cdot$ S. Schultze $\cdot$ M. Fenchel $\cdot$ H.-P. Schlemmer Department of Radiology (E010), German Cancer Research Center (DKFZ), Im Neuenheimer Feld 280, D-69120 Heidelberg, Germany e-mail: m.roethke@dkfz.de

T. H. Kuru • B. A. Hadaschik

Department of Urology, University Hospital Heidelberg,

Heidelberg, Germany

D. Tichy $\cdot$ A. Kopp-Schneider

Division of Biostatistics, German Cancer Research Center (DKFZ),

Heidelberg, Germany 